Article

\title{
Recent Research of Thorium Molten-Salt Reactor from a Sustainability Viewpoint
}

\section{Takashi Kamei}

Research Institute for Applied Sciences, 49, Tanaka-Oi-cho, Sakyo-ku, Kyoto 606-8202, Japan; E-Mail: hae00675@nifty.com; Tel.: +81-75-701-3164; Fax: +81-75-492-0679.

Received: 3 July 2012; in revised form: 20 August 2012 / Accepted: 24 August 2012 /

Published: 27 September 2012

\begin{abstract}
The most important target of the concept "sustainability" is to achieve fairness between generations. Its expanding interpolation leads to achieve fairness within a generation. Thus, it is necessary to discuss the role of nuclear power from the viewpoint of this definition. The history of nuclear power has been the control of the nuclear fission reaction. Once this is obtained, then the economy of the system is required. On the other hand, it is also necessary to consider the internalization of the external diseconomy to avoid damage to human society caused by the economic activity itself, due to its limited capacity. An extreme example is waste. Thus, reducing radioactive waste resulting from nuclear power is essential. Nuclear non-proliferation must be guaranteed. Moreover, the FUKUSHIMA accident revealed that it is still not enough that human beings control nuclear reaction. Further, the most essential issue for sustaining use of one technology is human resources in manufacturing, operation, policy-making and education. Nuclear power will be able to satisfy the requirements of sustainability only when these subjects are addressed. The author will review recent activities of a thorium molten-salt reactor (MSR) as a cornerstone for a sustainable society and describe its objectives and forecasts.
\end{abstract}

Keywords: thorium; molten-salt reactor; rare earth; externality; small modular reactor

\section{Introduction}

It is not suitable to apply the word "sustainability" to each technology or field by taking into account its origin. As is well known, the word "sustainability" was originally used in the report of UN's Brundtland committee to point out the importance of "sustainable development" [1]. Then the 
word "sustainability" came to be used in the discussions of global warming. Based on the report of the above committee, the meaning of the word "sustainability" is to achieve fairness between generations. This is to avoid the unfairness whereby future generations will be affected by the atmospheric temperature increasing, caused by artificial emissions of carbon dioxide from previous generations. It is also unfair that future generations become unable to utilize natural resources because of their consumption by previous generations. On the other hand, there is unfairness within the present generation, and the "south-north problem" is an extreme example. According to the above mentioned global warming, the case that the land area of pacific island nations will be decreased by the rise in sea level is such an example, because of the amount of carbon dioxide emitted from these countries is negligible compared to the amount from the U.S. and China, and so on. This can be described as unfairness between regions or unfairness within a generation. Thus, the word "sustainability" should be applied to the whole activity of humans and should not be used for some identified technology's continuity of utilization. In this manner, it is not suitable to discuss sustainability "of" nuclear power; we should discuss nuclear power "in" sustainability. In this paper, the author will describe how nuclear power is positioned within human society from the point of view of sustainability.

\section{Thorium}

\subsection{Direct Contributions and Subjects of Nuclear Power from a Viewpoint of Sustainability}

There are many aspects to discuss regarding the achievement of sustainability. One issue that each private company or nation cannot treat (since it is a cross-border phenomenon), is the reduction of artificially emitted carbon dioxide-within an international framework. About $40 \%$ of global scale carbon dioxide stems from the energy sector, and about $20 \%$ from transport [2]. Although there are many possibilities of application, the main purpose of nuclear power is to provide electricity and this will not change much in the future; it can be said that the value of nuclear power is to reduce the emission of carbon dioxide from the energy sector. It is also necessary to predict how much nuclear power can be implemented in order to quantitatively evaluate this value. In addition, what is important from the view of sustainability is how to evaluate the waste as an external diseconomy, which comes as a result of the benefit of electricity production [3]. The emission of carbon dioxide from fossil fuel power plants corresponds to this waste and its externality was studied by Pigou [4] because exhaustion gas seriously damaged people's health in England after the industrial revolution.

Radioactive waste contained in spent nuclear fuel is the most significant waste in the case of nuclear power. The refining tail at the site of uranium mining is also radioactive waste relating to nuclear power. Of course, there are other wastes resulting from the utilization of nuclear power, but we focus on the characteristic feature of radioactivity. The FUKUSHIMA accident, on 11 March 2011, revealed again that there is a possibility that commercial nuclear power plant releases emissions containing radioactive materials to an environment including the people living nearby. These radioactive materials are not necessarily "wastes" since they contain valuable materials which can be used as nuclear fuel. They will not be lost during normal operation of nuclear power plant. Therefore these radioactive materials can be determined as non-waste. However, taking into account that the release of radioactive materials will bring some late effects or hereditary effects to the public - especially near the accident 
site, which is far from the area of usage of electricity emerging from this nuclear power plant, - this can be determined as unfairness within a generation or unfairness between regions, being the remaining subject of nuclear power from the viewpoint of sustainability. This subject can be overcome by the improvement of safety in nuclear power plants.

The nuclear security summits held in April 2010 and March 2012 appealed to the U.S. and Russia not only to dismantle existing nuclear weapons, but also promote the peaceful use of nuclear power [5]. As long as nuclear power relies on the nuclear fission reaction, it is impossible to avoid fissile isotopes which can be used as nuclear weapons. This is an essential difference between nuclear power and other energy sources. The former Swedish Prime Minister, Mr. Palme, made a speech at the United Nations in which he said that war is the main activity which destroys sustainability [6]. In this regard, it is indispensable to improve the resistivity of nuclear proliferation from the view of sustainability when we utilize nuclear power, even for peaceful purposes.

\subsection{Indirect Contribution of Nuclear Power to Sustainability}

As mentioned above, it is necessary to reduce the emission of carbon dioxide from the transport sector. Therefore, electric vehicles (EVs) which do not emit carbon dioxide during operation, or hybrid vehicles (HVs), whose carbon emissions are less than ordinary gasoline cars are now being developed by many car companies around the world. Some have already been supplied to the market. The most apparent difference of these low-carbon vehicles from widely used combustion engine cars is that they use an electric motor as the driving mechanism. Also, for the material used in manufacturing the permanent magnet, core components of the electric motor are "rare earths" [7]. Neodymium and dysprosium supplement steel in order to enhance the magnetic force or thermal resistivity. The difficulty in obtaining rare earths has become a serious problem in the supply chain of the high-technology industry in recent years. The essential reason is that nobody has a countermeasure for thorium, which is a by-product of rare earth production.

The issue of thorium is an external diseconomy relating to rare earth production. Thus, internalization has to be achieved; otherwise the thorium as waste is thrown into the environment and causes a societal hazard. The ways of internalizing this thorium in human society can be summarized simply in two steps. The first is to store and manage thorium not as valueless waste, but as a material hazardous to the public. In this case, it may be adequately undertaken at the beginning of such an operation, but would be incorrect if done later as people tend to forget the existence of "hidden" issues. It is also not easy to accrue financial support to store this unwelcomed material. The second way is to store thorium but as a valuable material. The largest value of thorium is as an energy source of nuclear fuel. The method to "internalize" is described in detail in a previous paper [8]. A proposal for the international storage of thorium is named as "The Bank (Thorium Energy Bank) [9]". A financial mechanism is also mentioned in the recent paper by establishing the "OREEC (Organization of Rare-Earth Exportation Countries) [10]". Anyway, it is pointed out here in the context of this section that radioactive thorium exists as externality in a flow of achieving sustainability which can be combined with nuclear power and this will remain as an element of obstruction of sustainability unless thorium is adequately taken care. Incidentally, there are numerous products which use rare earth. Cerium is used to enhance resistivity against ultraviolet rays of grew 
resin which is used to fix solar module's glass and photovoltaic cell. Neodymium and dysprosium are also used for dynamo of windmills since the mechanism of dynamo and electric motors is basically same. These two elements are indispensable for mobile phones. Cerium is also used as a polisher of liquid crystal panels. Gadolinium is quite important for nuclear power because it is used in a control rod as a neutron absorber.

\subsection{Characteristics of Thorium from the View of Sustainability}

As we discussed in the previous sub-sections, it can be said that nuclear power has to achieve: (1) a countermeasure to reduce workload of radioactive waste; (2) improved safety; (3) enhanced nuclear proliferation resistivity; and (4) a way to take care of thorium occurring as a byproduct of rare earth refining in spite of it not being directly related to existing nuclear power. In this sub-section, we will discuss how these requirements will be cleared in the technological system of nuclear power from a sustainability viewpoint.

Firstly, the forth requirement would best be stored and managed under the international framework proposed as "The Bank". Otherwise, there is no guarantee that thorium would be adequately stored responsibly. Its financial governance should also be prepared under the framework of "The OREEC". In any case, with regard to storage of some material, there is a hope that it becomes of value in the future; i.e., it is assumed that thorium will be used as a nuclear fuel but is correctly stored and managed even in the case of no utilization.

This also answers some requirements of (1) to (3) which are connected with the present nuclear power system. The first requirement will be improved once thorium is used as fertile material due to its generating uranium-233's minor production amount of transuranic nuclide. Especially the very amount of produced americium and curium contributes to reduce radioactive toxicity of radioactive waste [11]. It should be mentioned here that this is a comparative discussion to the radioactive waste of uranium fuel and we should understand that there are several isotopes whose half-life is still longer than 10 thousand years.

The second requirement will not be achieved only by applying thorium as nuclear fuel. For example, the accidental sequence which happened in FUKUSHIMA would happen even if the plants used thorium fuel rods. However, the use of molten-salt reactor which will be discussed in the next section is expected to greatly enhance safety [12]. The first requirement can be achieved qualitatively in almost any kind of reactor due to common characteristics such as fertile thorium. For the case of using thorium in molten-salt reactor, the amount of whole radioactive waste will be reduced because this type of reactor does not use frequently replaced nuclear fuel rod. The third requirement will be satisfied naturally as long as the total system is adequately designed. Note that uranium-233 generated from thorium is a fissile isotope and its significant quantity is defined as $8 \mathrm{~kg}$ by the IAEA (International Atomic Energy Agency) [13]. In this regard, there remains the possibility of nuclear proliferation, theoretically if pure uranium-233 is obtained from thorium fuel cycle [14]. On the other hand, it should be pointed out that the accompanying strong gamma rays from thallium-208, being the daughter nuclide of thorium, makes it practically difficult to steal uranium-233 for the use of making nuclear weapons. Thus, it can be said that thorium utilization contributes to achieving sustainability and the effect will be enhanced with a collaborative use of a molten-salt reactor. 


\subsection{Characteristics of Thorium from the View of Nuclear Power}

Thorium is fertile and thus can contribute as an option of nuclear power toward sustainability by producing electricity with little emission of carbon dioxide. In addition to the advantages mentioned in the previous section, it is often said that thorium has attractive features, such as: (1) abundant resources about three times larger than uranium; (2) widely available resources in the world; (3) large $\eta$ value of uranium-233 obtained from neutron absorbed thorium in the thermal region; and (4) availability of fissile breeding in thermal neutron region; and so on.

Above features (1) and (2) are indicative of the expectation that thorium will be obtained with a low price once thorium is used as nuclear fuel [15]. It is not necessarily wrong as an elementary overall prediction; however, it still should be noted that the evaluation is not suitable to predict future availability of thorium nuclear power. In order to evaluate availability of thorium utilization, we must take into account thorium's nucleus characteristics being different from uranium. That is to say, thorium does not have its own fissile isotope. (Although it was not an academic program, on 13 June 2012 NHK broadcast [16] introduced a history of Japanese nuclear power development, stating that thorium was strongly considered in Japan in 1950s, i.e., the very beginning of Japanese nuclear history, but such a trial was conducted by a fast breeder reactor using plutonium because thorium was not useful for nuclear weapons. This short narration indicates that thorium cannot be used for nuclear fuel only by itself and thorium needs to be used in conjunction with a fissile material such as plutonium or a highly enriched uranium-235 or uranium-233. There was no stipulation that enriched uranium-235 is better for use with uranium-238. Further there was no plutonium and uranium-233 available in the 1950 s as it can only be obtained from artificial radiation in a nuclear reactor. This is a typical example of Japanese media explaining thorium).

In order to evaluate how much thorium nuclear power contributes to sustainability as a low-carbon energy source, its implementation capacity must be known quantitatively. Such an evaluation considering thorium's limited condition of having no fissile isotope cannot be seen except in the author's work [17,18]. Consequently, available implementation capacity of thorium fuel cycle using molten-salt reactor supported by plutonium supply from uranium fuel cycle will be about 392 GW by 2050 [19]. This amount will correspond to only a small percent of primary energy. Uranium-233 obtained as a result of thorium usage is also included as fissile material in this calculation. If weapon grade plutonium from predicted dismantled nuclear weapons is supplementarily fed to implement thorium molten-salt reactor, its contribution will be about $10 \mathrm{GW}$ [18]. These simulations were carried out to evaluate the role of fissile material for starting thorium nuclear fuel cycles and no significant considerations of industrial background such as manufacturing capacity of molten-salt reactors, reprocessing of spent nuclear fuel of uranium fuel cycle, reprocessing of spent liquid fuel of thorium molten-salt reactor were involved. Therefore, it can be estimated that the above value is rather optimistic.

Though there is some expectation that a thorium market will be formed once thorium is used as an energy source, it should be mentioned that such a market to trade thorium will not be true taking into account both the limited available implementation capacity of thorium nuclear power controlled by limited supply of fissile material externally from the uranium fuel cycle and the large production of thorium as a byproduct of rare earth refining. This is because production of thorium is much larger 
than the predicted required amount based on the above simulation [8]. This means that there will be no motivation in the business sector to develop "new" "thorium" mining because there are huge amounts of excess thorium. In addition, this excess thorium is shared by only four countries, i.e. India, the U.S., Australia and China. If these four countries supply their excess thorium free of charge, development of thorium mining carried out by the private sector will be terminated based simply on market mechanisms. Therefore, we should not make too optimistic a prediction for thorium resource development.

Of course, development of thorium nuclear power itself has its own rational value in spite of the above considerations. For example, India is known as the country that has a unique developmental program of thorium utilization. India at first developed a heavy water reactor based on the technology of CANDU and used uranium for more than 40 years. This is known as the first stage. The purpose of this stage is to accumulate plutonium which can be used as fissile material with national abundance of thorium. In the second stage, several fast breeder reactors which use both plutonium and thorium for fissile and fertile materials respectively have been in operation in recent years. This is still not the final formation of India's nuclear power as India will then, in the third and final stage, utilize the uranium-233 which is generated in the above fast breeder reactors. It is often said that India is developing an advanced heavy water reactor for the utilization of uranium-233 and thorium in the third stage but this describes only one aspect of the whole. There are other candidates being considered and molten-salt reactor is one of them. India's nuclear developmental scenario is shown in Figure 1 [20].

Figure 1. India's developmental scenario of nuclear power (red outline by the author).

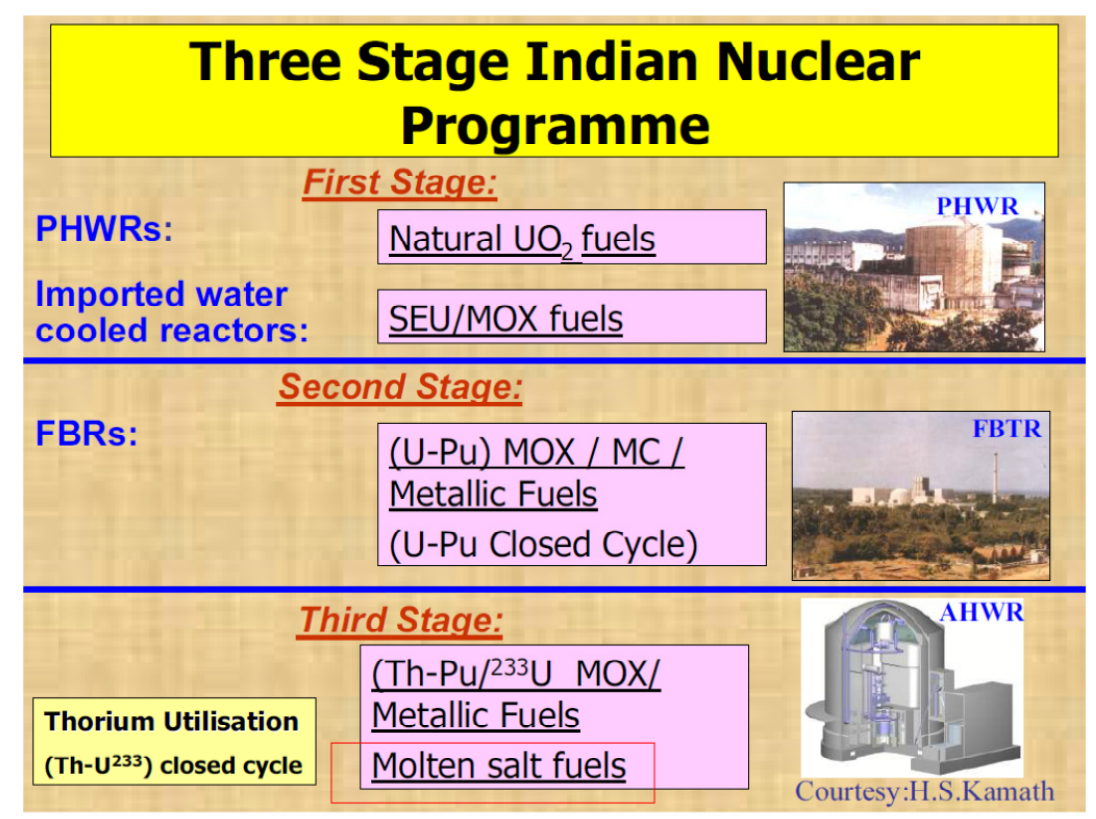

China has already started using thorium fuel rod partially in CANDU at the Qinshan nuclear power plant. China also decided to start development of a thorium molten-salt reactor in January 2011. A thorium fuel-working group was formed in atomic energy society of Japan in May 2010 to study thorium utilization in light water reactors and fast reactors with solid fuel rod. There are several activities to develop molten-salt reactor for using thorium. 


\section{Molten-Salt Reactor}

\subsection{Advantages of Molten-Salt Reactor}

Molten-salt reactor is a nuclear reactor whose basic concepts were proposed in the 1950s in the U.S. [21]. The reactor is operated by using liquid fuel instead of solid fuel rod. Its liquid medium is molten-salt. Fissile and fertile materials are dissolved into this liquid phase. The liquid medium also acts both as solvent of the fission product and as a heat transferring medium. Molten-salt reactor experiment (MSRE) was designed and was successfully operated at Oak Ridge National Laboratory (ORNL) for four years from 1965. This is the newest operated molten-salt reactor and there is no constructed molten-salt reactor.

The history of nuclear reactors was weighted on the ability of "breeding" to obtain more reproduced fissile material than its consumption because of the extremely small amount of uranium-235 there is in nature and lack of fissile isotope in thorium. Therefore, it is rational that a fast breeder reactor with plutonium was the major research and developmental target in the world until the 1990s because $\eta$ value of plutonium is the highest in fast neutron region. Molten-salt reactor was recognized to have an excellent breeding feature and molten-salt breeder reactor (MSBR) was designed after the success of MSRE. MSRE was constructed to confirm characteristics of configuration and material proposed for molten-salt reactor. Thus, thorium itself was not used and only fissile materials were fed for sustaining chain reaction. The high availability of breeding in MSBR could be achieved by (1) using thorium as fertile material so as to produce uranium-233 which has large $\eta$ value in thermal region; (2) high efficiency of neutron usage avoiding existence of gaseous neutron poison such as xenon from reactor core; (3) online reprocessing facility to remove fission product (and protactinium as long as thorium is used as fertile material) enabling highly effective use of neutron.

Molten-salt reactor is expected to have high thermal efficiency due to its high operation temperature being about $700{ }^{\circ} \mathrm{C}$ at the exit of primary circuit from reactor core. This reactor also exhibits high inherent safety. For example, freeze valve placed just below the reactor vessel automatically opens when fuel salt reaches a high temperature. Liquid fuel is automatically drained to a lower drain tank merely by gravity. In the case of station black out which occurred at FUKUSHIMA, the freeze valve correctly works because molten-salt is frozen by an electrically operated fan. That is to say, the loss of electricity at the reactor automatically stops this fan and frozen salt melts. Moreover, in the case of leakage of fuel salt from reactor vessel or pipes, the molten fuel will soon freeze due to its high frozen point being about $450{ }^{\circ} \mathrm{C}$. Its economy is also attractive which is achieved by eliminating the use and fabrication of fuel rods. Bottleneck in supply chain of manufacturing present light water reactor to fabricate pressure vessel does not occur in molten-salt reactor. This means that molten-salt reactor has a possibility to establish a new nuclear power industry which is not connected to the Japan Steel Works which provides pressure vessels [22]. These advantages will be especially effective to realize small modular reactor (SMR). 


\subsection{Remaining Subjects of Molten-Salt Reactor}

Though there are many advantages in the concept of molten-salt reactors, there are still some remaining many subjects which have to be cleared before its commercialization. For example, MSBR focused on enabling high conversion ratio. MSBR is a thermal neutron reactor thus MSBR was designed to have small leakage of neutron from reactor core. MSBR adopted graphite as moderator. As a result, internal region of reactor vessel is filled with moderator graphite. Volume of solid graphite does not expand significantly when temperatures increase, thus the ability of moderation of neutron is not lost. On the other hand, volume of liquid fuel can expand against temperature rise. This contributes to coarse ability of moderation of neutron. However, in the case of MSBR which has large volumetric ratio of solid phase by graphite, total temperature reactivity coefficient can be positive. Original evaluation carried out by ORNL indicated that temperature reactivity coefficient becomes negative but the value has been recalculated in 2005 to have a positive value [23]. In the explanation of molten-salt reactor in Wikipedia, a conceptual illustration is shown which indicates the use of graphite moderator [24]. This illustration is originally made for generation IV international forum (GIF). In contrast to this, the web site of GIF apparently claims that present concept of GIF's molten-salt reactor does not use graphite moderator by showing a large red cross [25].

Furukawa pointed out the difficultness of online reprocessing unit considered in the design of MSBR and proposed a molten-salt reactor named "FUJI series" which enables conversion ratio to be 1, even by eliminating the online reprocessing unit [15]. Designing policy of FUJI series is simple: (1) no use of online reprocessing; (2) thermal spectrum reactor; and (3) reduce leakage of neutron from reactor core. As a result, volumetric ratio of solid graphite moderator was kept high in reactor core for achieving the conversion ratio of 1 . Furukawa revealed that FUJI has negative temperature reactivity coefficient [15] but Hirose implied that the value would be positive [26].

The question of temperature reactivity coefficient can be solved by taking other criteria of designing molten-salt reactor. For example, the value will become small if neutron leakage from reactor core is increased. In this case, conversion ratio will be somewhat small. Selecting fast spectrum neutron instead of thermal spectrum will be another possibility. This can be done by eliminating graphite moderator from the core. French MSFR [27] and Russian MOSART [28] correspond to this approach.

Most of the proposed designs of molten-salt reactor are targeted to be a large capacity power reactor. Though there were several detailed differences in reactor core designs, the basic configurations as a power system were the same. That is to say: (1) fission reaction happens in the core; (2) generated thermal energy heats up the surrounding molten-salt fuel; (3) hot liquid fuel is transferred from reactor core to intermediate heat exchanger through external pipe; (4) thermal energy is transferred to secondary coolant via thin wall of intermediate heat exchanger; (5) cooled molten-salt fuel is pumped back to go into the reactor core again. The path where molten-salt fuel goes around is called the primary circuit. Most of the other remaining subjects relating to molten-salt reactor are caused by this basic configuration. That is to say, these subjects are caused by the radioactive materials including fission products leaving the reactor core and circulating in the primary circuit. For example, the main subjects are: (1) increase of radiation background around primary circuit; (2) loss of delayed neutrons due to the flowing out of precursor toward external pipe; (3) erosion of thin thickness external pipe by direct contact of molten-salt containing many different kind of fission products especially in the region 
of intermediate heat exchanger; (4) transportation of tritium generated by neutron capture of lithium- 6 from molten-salt fuel to secondary coolant because it is easy for tritium going through metal material; and (5) uncertainty of availability of containing fission product inside of primary circuit where there are pumps. Though many other small problems are solved, the safety of the molten-salt reactor will never be achieved unless at least these main problems are solved. In order to help understanding of these difficulties of molten-salt reactor, the technical way involved in the present light water reactor taking care of similar questions will be shown in Figure 2.

Figure 2. Nuclear reactions and radioactive materials contained in light water reactor.

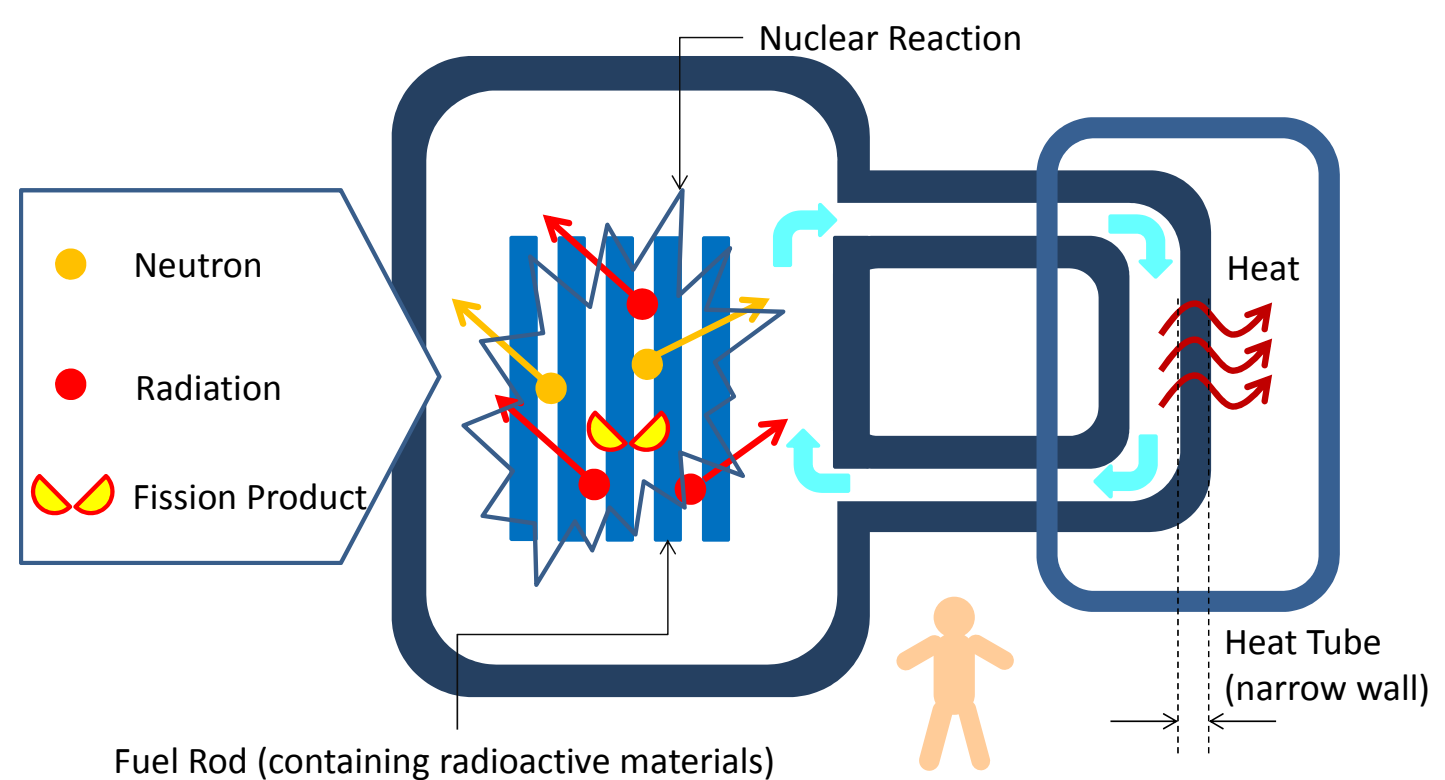

Maintenance (low exposure)

As shown in Figure 2, nuclear fuel exists only in the center of reactor core and nuclear reaction also happens in this region in the case of the light water reactor. Newly generated radioactive materials are also kept in this region namely inside the nuclear fuel rod. Though high radiation occurs, it is limited around the reactor vessel. Thus, theoretically, radiation background around primary circuit does not become high. The delayed neutrons, which play an important safety role, occur only in the reactor core because precursors are kept in the nuclear fuel pellets. Thermal heat generated in the reactor core is transferred via thin wall of steam generator (in the case of pressurized water reactor) to a secondary loop. Though often a small pinhole is shaped on this thin wall of steam generator, basically no radioactive materials are contained in the coolant of primary circuit. Tritium is also produced in light water reactor but the amount is much smaller than in the case of the molten-salt reactor which utilizes lithium as a medium.

Subjects relating to molten-salt reactor are illustrated in Figure 3. Whereas, in the case of light water reactor, nuclear reactions including decay happen not only inside reactor core but also in pipes of primary circuit. Thallium-208 goes through pipes of primary circuit and thus radiation around these pipes increases. Exposure to radiation will be greater than in the case of light water reactor. Though this tendency depends on the length of pipes, many delayed neutrons are lost outside of reactor core and the safety margin of nuclear reaction control is decreased. Of course, these lost neutrons are not used for converting thorium to uranium-233. Even though a modified Hastelloy $\mathrm{N}$ has been developed 
as material of molten-salt reactor which improved resistivity of erosion by tellurium as fission product, its reliability under operational conditions has to be confirmed, especially for a thin wall instrument such as intermediate heat exchanger. These problems are common in previously proposed molten-salt reactors such as FUJI.

Figure 3. Nuclear reactions and radioactive materials contained in molten-salt reactor.

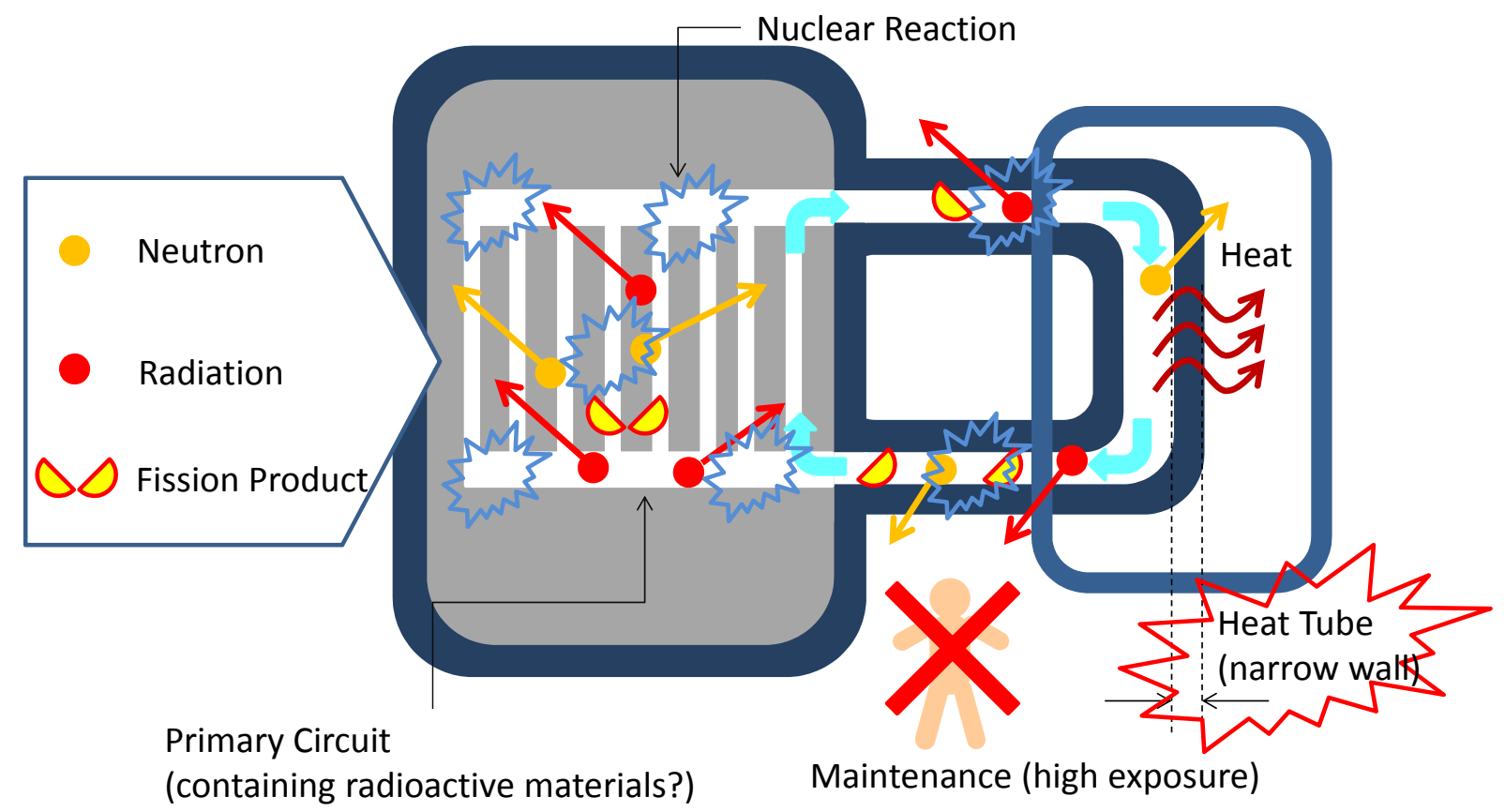

\subsection{Development of "Internal Circulating and Surface Heat Transferring Molten-Salt Reactor"}

Requirements of nuclear power from the view of sustainability can be summarized as follows. They are: (1) reduction of waste including radioactive materials; (2) enhancement of nuclear non-proliferation; (3) high safety; and (4) availability of small capacity with enough high economy. In addition (5) availability of load-following operation and (6) eliminating bottle neck of supply chain such as Japan Steel Works having more than an $80 \%$ share of pressure vessel production have to be satisfied. Small capacity reactor can be of course done in a scheme of light water reactor but economy is not necessarily high because of the production and exchange of fuel rods. For satisfying requirement (1), thorium was selected as fertile material and basic concept of molten-salt reactor was chosen. Concept of molten-salt reactor also enables to have small size of the reactor with acceptable economy. Bottleneck in supply chain of producing large-scale light water reactor can be eliminated by adopting molten-salt reactor which has low inner pressure. Since the reactor size is small, there may be relatively small efficiency of neutron usage or large amount of neutron loss. It means that small molten-salt reactor tends to have a small conversion ratio. Thus, the value of conversion ratio is not considered to have high priority in this study. Even though molten-salt reactor is adopted as fundamental concept, the previously proposed design using primary circuit for heat transfer such as FUJI does not solve molten-salt reactor's engineering problems mentioned above. That is to say, the heat generated in reactor core is transferred to external heat exchanger via primary circuit by using fuel salt. Therefore, the author is developing a new concept of molten-salt reactor to overcome these 
subjects by eliminating the function of heat transfer via primary circuit. The concept's structural features are illustrated in Figure 4. A patent for this design of "internal circulating and surface heat transferring molten-salt reactor" is in process (Request number 2012-122679 in Japan).

Figure 4. Conceptual design of internal circulating and surface heat transferring molten-salt reactor.

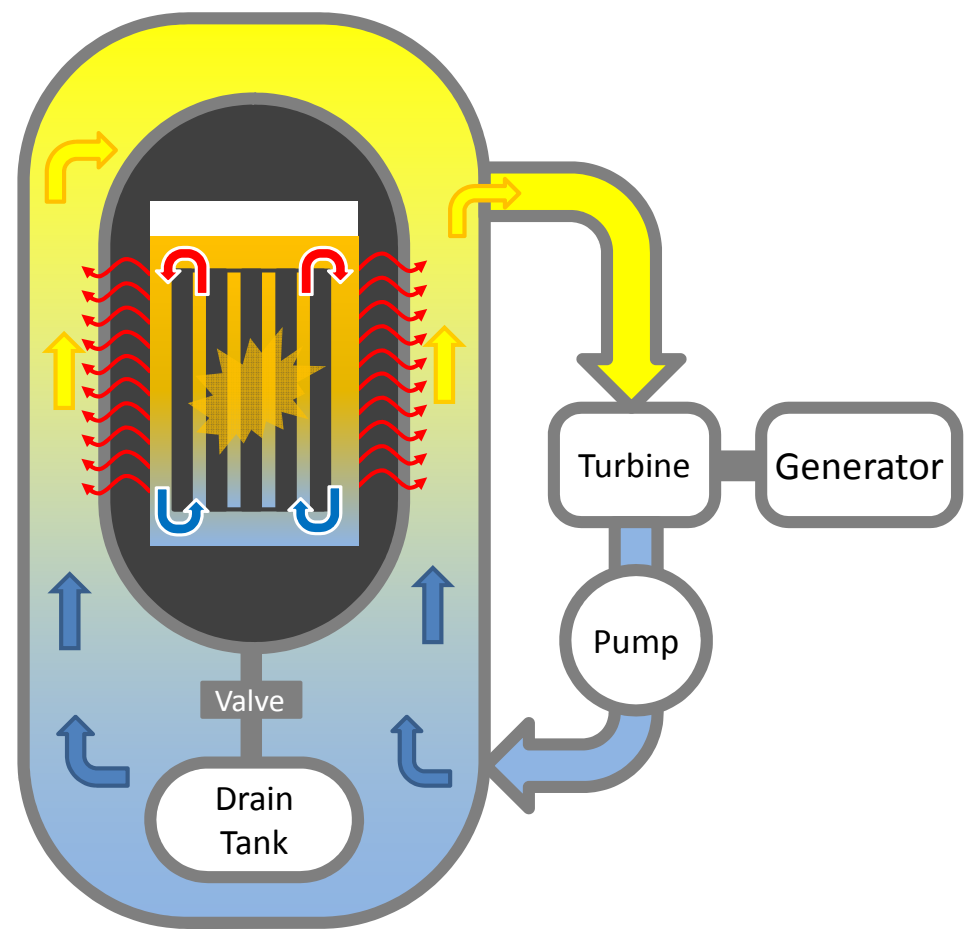

In this new concept, thermal energy generated in the core is transferred by fuel salt itself. The fuel salt flows upward in the core and then moves in a peripheral direction at the top of the core. It goes into the gap formed by graphite moderator and peripheral reflector. The fuel salt transfers its heat to peripheral reflector while it goes down this gap. The transferred heat moves by thermal conduction within both the peripheral reflector and its surrounding reactor vessel. Finally, the heat is moved by coolant which flows outside of the reactor vessel. Namely, the fuel salt does not go out of reactor vessel via narrow tubes having thin wall thickness optimized for high efficiency of heat transfer.

Graphite is used in the core as moderator. Thus, this concept is a thermal reactor. Temperature reactivity coefficient can be negative by the limiting small size of the reactor. It is estimated that leakage of neutrons will be increased due to such a small reactor resulting neutron radiation outside of reactor vessel and essentially low efficiency of neutron. Therefore, external reflector is equipped outside of the reactor vessel to reduce neutron loss as shown in Figure 5. This external reflector is movable toward axial direction. Thus, it enables to actively control the ratio of neutron reflection. This keeps temperature reactivity coefficient to be negative value. In contrast to the previously proposed molten-salt reactors which lose delayed neutrons in pipes of primary circuit because of no reflection around pipes, this new conceptual design can keep the delayed neutron within the reactor vessel. Molten-salt reactor has an ability of load following but its time-constant is more than 10 minutes [29]. This value must be much shorter if the small molten-salt reactor proposed here is used 
in a small-scale grid. It is expected that this function of active control of neutron reflection will improve the ability of load following operation.

Figure 5. Schematic diagram of internal circulating and surface heat transferring molten-salt reactor.

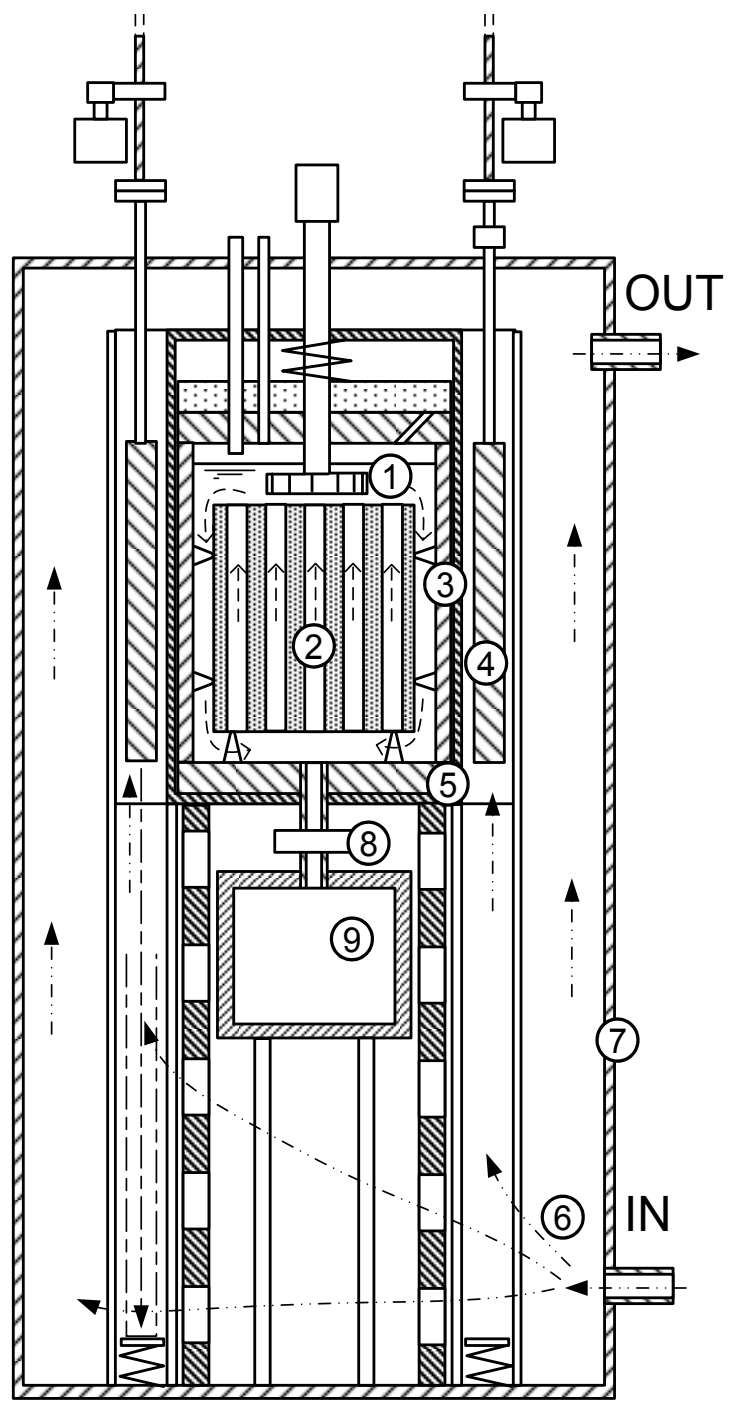

(a) Side View
1. Molten-salt fuel (internal circulation)

2. Reactor core with graphite moderator

3. Peripheral reflector

4. External reflector

5. Reactor vessel

6. Coolant

7. Containment vessel

8. Freeze valve

9. Drain tank

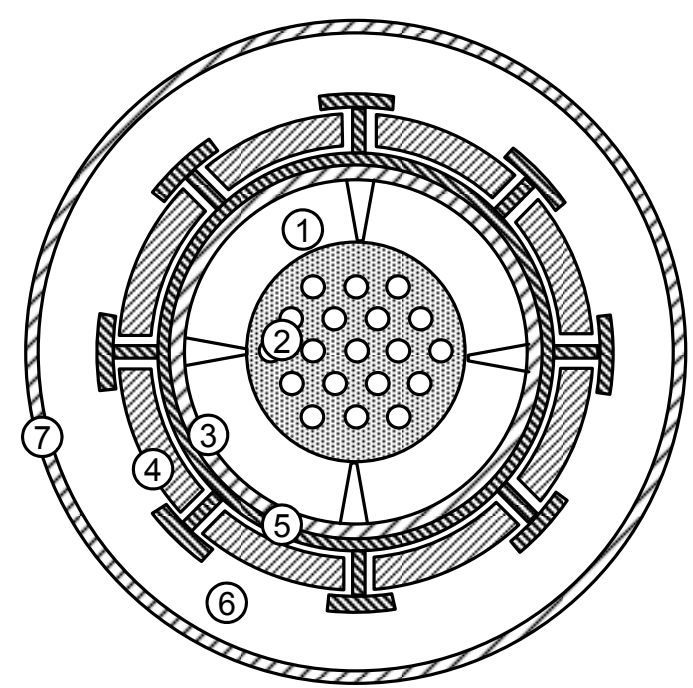

(b) Top View

Ceiling and bottom of the reactor core are also covered by neutron reflectors, therefore fuel salt does not directly contact with reactor vessel. This non-direct contact of fuel salt with reactor vessel enables avoiding the mass-transfer phenomena caused by solubility difference of metallic components to salt at hot part and cold part. It is also expected that most of tritium produced in a reaction between lithium-6 and neutron will be kept inside the reactor vessel because there is little contact of fuel salt and vessel. Noble gases such as xenon which act as neutron poison do not dissolve in the fuel salt and are kept in the upper space of reactor vessel. From these features, this conceptual design can be expressed as an "internal circulating and surface heat transferring" molten-salt reactor. This design can equip high safety by eliminating pipes of primary circuit.

Generally speaking, the purpose of the primary circuit is only heat transfer and thus it has a large enough capacity for heat removal. It is imagined that the capacity of thermal generation in the 
proposed design with no primary pipes, will be small. Although this design does not require a large capacity because the motivation of this study is to realize a small reactor with high safety yet remaining economical, it is necessary to evaluate upper limit of thermal generation from an engineering point of view. Neutronics behavior of this design has been confirmed in a preliminary stage of discussion and now its detailed study is being carried out. The results will be reported in the near future. In this paper, upper limit of thermal capacity of this reactor is demonstrated as below.

In this design, there is a molten-salt as hot fluid in the center of reactor vessel and solid peripheral reflector and reactor vessel surround the fuel salt as cylinders. Cold working fluid flows outside of reactor vessel removes heat. Though there is a solid moderator made of graphite in the fuel salt, it is assumed that the core is filled with homogeneous fuel salt. Then the system can be treated simply as a multi-layered cylinder. Calculation model of heat transfer is illustrated in Figure 6.

Figure 6. Calculation model of heat transfer.

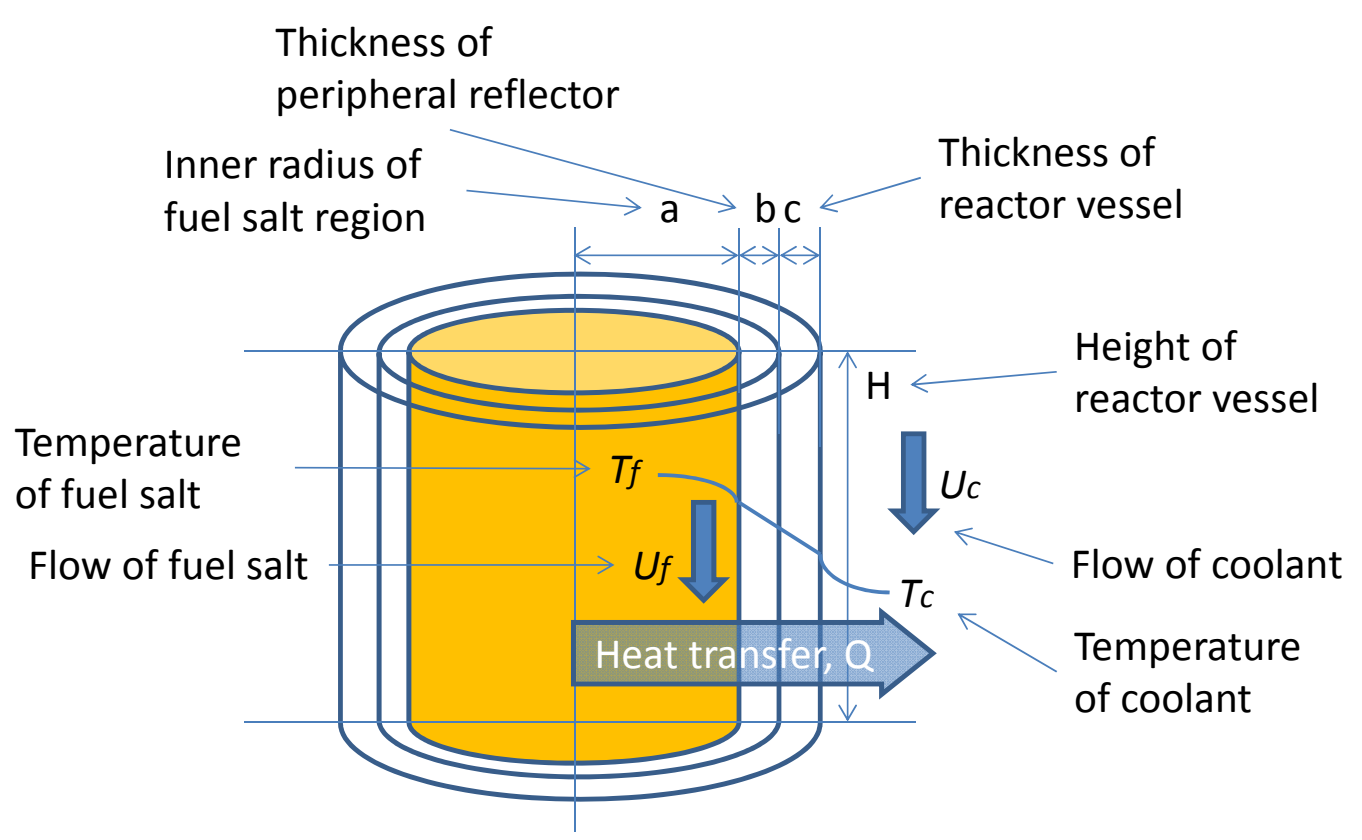

Amount of heat transfer $Q$ from multi-layered cylinder can be expressed as Equation (1).

$$
Q=\frac{T_{f}-T_{c}}{R}
$$

here, $R$ is total thermal resistance, $T_{f}$ is temperature of fuel salt, $T_{c}$ is temperature of coolant. $R$ can be expressed by using thermal resistance of fuel salt $\left(R_{f}\right)$, that of peripheral reflector $\left(R_{r}\right)$, that of reactor vessel $\left(R_{v}\right)$ and that of coolant $\left(R_{c}\right)$ as shown in Equation (2).

$$
R=R_{f}+R_{r}+R_{v}+R_{c}
$$

Each thermal resistance can be expressed as Equations (3-6).

$$
R_{f}=\frac{1}{2 \pi a H h_{f}}
$$




$$
\begin{gathered}
R_{r}=\frac{b}{2 \pi a H k_{r}} \\
R_{v}=\frac{c}{2 \pi(a+b) H k_{v}} \\
R_{c}=\frac{1}{2 \pi(a+b+c) H h_{c}}
\end{gathered}
$$

here, $a, b$ and $c$ are radius from center of reactor to peripheral reflector, thickness of this reflector and thickness of reactor vessel, respectively. $H$ is height of reactor vessel. $k_{r}$ and $k_{v}$ are thermal conductivity of peripheral reflector and reactor vessel. $h_{f}$ and $h_{c}$ are heat transfer coefficient of fuel salt and coolant. $h_{f}$ and $h_{c}$ can be expressed as Equations $(7,8)$.

$$
\begin{aligned}
& h_{f}=\frac{N u k_{f}}{L} \\
& h_{c}=\frac{N u k_{c}}{L}
\end{aligned}
$$

here, $L$ is representative length and $N u$ is Nusselt number. In this study, it is assumed that the reactor vessel has large diameter and the surface can be treated as flat plate. $N u$ is determined by condition of flow. Condition of fuel salt can be evaluated by using Equation (9).

$$
R e=\frac{U_{f} L}{v}
$$

here, $R e$ is Reynolds number. $U_{f}$ is superficial fuel salt velocity. $v$ is kinematic viscosity of fuel salt. In this study, Flibe ( $\left.\mathrm{LiF}-\mathrm{BeF}_{2}-\mathrm{ThF}_{4}-\mathrm{UF}_{4}: 71.76,16,12,0.24 \mathrm{~mol} \%\right)$ is considered as fuel salt. Hastelloy $\mathrm{N}$ is used as reactor vessel's structural material. Characteristics of these materials are summarized in Table 1.

Table 1. Characteristics of materials considered in this study.

\begin{tabular}{cc}
\hline Item & Value \\
\hline$\rho$ (Flibe) & $2,270 \mathrm{~kg} / \mathrm{m}^{3}$ \\
$k_{f}$ (Flibe $)$ & $1.2 \mathrm{~W} / \mathrm{mK}$ \\
$C_{p}$ (Flibe) & $2,383 \mathrm{~J} / \mathrm{kgK}$ \\
$v$ (Flibe) & $1.15 \times 10^{-5} \mathrm{~m}^{2} / \mathrm{s}$ \\
$k_{r}$ (Graphite $)$ & $100 \mathrm{~W} / \mathrm{mK}$ \\
$k_{v}$ (Hastelloy $\left.\mathrm{N}\right)$ & $18 \mathrm{~W} / \mathrm{mK}$ \\
\hline
\end{tabular}

Re of fuel salt becomes 8696 from Equation (9) by considering that the representative length $L$ is $1 \mathrm{~m}$ corresponding to height of reactor vessel and superficial velocity $U_{f}$ is $0.1 \mathrm{~m} / \mathrm{s}$. The condition of fuel salt on a flat plate is laminar flow because above $R e$ is smaller than $3 \times 10^{5}$. The flow is forced convection. Then Equation (10) can be used to obtain $N u$ for fuel salt.

$$
N u=0.664 R e^{1 / 2} \operatorname{Pr}^{1 / 3}
$$


Here $\operatorname{Pr}$ is Prandtl number and determined by Equation (11). Its value becomes 52.

$$
\operatorname{Pr}=\frac{v}{\alpha}=\frac{v \rho C_{p}}{k}
$$

Then $N u$ becomes 228 and $h_{f}$ becomes 273 .

There are many candidates of coolant such as water, liquid metal, molten-salt and gaseous medium. In our present studies, helium is considered because this concept is aimed at realising a small and compact reactor. Characteristics of helium depend strongly on pressure and temperature. Density of helium can be obtained by Equations (12-14). Thermal conductivity of helium can be obtained by Equations $(15,16)$. Viscosity of helium can be obtained by Equations $(17,18)$.

$$
\begin{gathered}
\rho=\frac{P}{R T}-\rho^{2} B(T) \\
B(T)=4.5 \times 10^{-4}+\frac{5.42}{(1,890+T)} \\
\rho=\frac{-1+\sqrt{1+4 B(T) \frac{P}{R T}}}{2 B(T)} \\
k_{0}=2.97 \times 10^{-3} T^{0.69}+\frac{9.23 \times 10^{9}(T-273.16)}{(T-273.16)^{5}+4.29 \times 10^{14}} \\
k=k_{0}+2.33 \times 10^{-4} \rho+2.39 \times 10^{-6} \rho^{2} \\
\mu_{0}=3.78 \times 10^{-7} T^{0.69}+\frac{5 \times 10^{-7}}{0.52+\frac{T}{569.6}} \\
\mu=\mu_{0}+2.67 \times 10^{-10} \rho^{2}
\end{gathered}
$$

$R$ is gas constant being $2.07723 \times 10^{-3} \mathrm{MPa} / \mathrm{kgK}$. Pressure and temperature of coolant are $4 \mathrm{MPa}$ and $873 \mathrm{~K}$, respectively. Then density of helium becomes $2.2 \mathrm{~kg} / \mathrm{m}^{3}$, thermal conductivity becomes $0.33 \mathrm{~W} / \mathrm{mK}$ and viscosity becomes $4.06 \times 10^{-5} \mathrm{~kg} / \mathrm{ms}$. Kinematic viscosity can be obtained by Equation (19) and the value is $1.85 \times 10^{-5} \mathrm{~m}^{2} / \mathrm{s}$.

$$
v=\frac{\mu}{\rho}
$$

Specific heat capacity of helium is 5192 and then $\operatorname{Pr}$ of helium becomes 0.64 . Re of coolant helium becomes $5.4 \times 10^{5}$ for its superficial velocity being $10 \mathrm{~m} / \mathrm{s}$. Flow condition of coolant is turbulent flow because above $R e$ is larger than critical value between laminar flow and turbulent flow being $3 \times 10^{5}$. $N u$ of coolant helium can be obtained by Equation (20). This equation is also for a flow on flat plate but the flow is turbulent. In this case, the value is 1231 .

$$
N u=0.037 \operatorname{Re}^{0.8} \operatorname{Pr}^{0.33}
$$


Heat transfer coefficient of helium can be calculated to be 405 from Equation (8). Each thermal resistance can be obtained as below by assuming that reactor vessel's outer radius is $0.5 \mathrm{~m}$, thickness of peripheral reflector is $0.1 \mathrm{~m}$, thickness of reactor vessel is $0.01 \mathrm{~m}$ and height of reactor vessel is $1 \mathrm{~m}$.

$$
\begin{aligned}
& R_{f}=1493 \times 10^{-6} \\
& R_{r}=408 \times 10^{-6} \\
& R_{v}=181 \times 10^{-6} \\
& R_{c}=786 \times 10^{-6}
\end{aligned}
$$

It can be seen from the result that the governing factor of heat transfer in this newly proposed reactor is mainly by thermal resistance of fuel salt. Then thermal resistance of helium coolant and that of peripheral reflector are large. Thermal resistance of reactor vessel can be improved by reducing its thickness but it should not be small from a mechanical intensity point of view. It is not necessary to seriously consider this value as far as it is smaller than other resistances.

It is not necessary to improve thermal resistance of peripheral reflector because it is smaller than that of helium coolant, too. Thickness of peripheral reflector affects neutron behavior. If this thickness is small, the amount of neutrons going through the vessel will be increased. Even though these neutrons can be returned to reactor by external reflector, neutron radiation of reactor vessel will be enlarged. Therefore, thickness of peripheral reflector must be evaluated from both considerations of heat transfer and neutronics. Detailed discussion of this will be reported in the near future.

Fuel salt existing in the reactor core has a possibility of phase change. Thus, temperature of fuel salt has to be kept lower than boiling point of the fuel, i.e., $1703 \mathrm{~K}$. Thermal power corresponding to above conditions is $289 \mathrm{~kW}$. Melting point of graphite being $3823 \mathrm{~K}$ is large enough compared to boiling point of fuel salt.

Thermal resistance depends on surface area of heat transfer. Therefore, thermal resistance can be

\begin{tabular}{|c|c|c|c|c|c|c|}
\hline \multirow{2}{*}{$\frac{U_{f}}{a+b+c, H}$} & \multicolumn{4}{|c|}{$0.1 \mathrm{~m} / \mathrm{s}$} & \multirow{2}{*}{$\frac{1 \mathrm{~m} / \mathrm{s}}{1 \mathrm{~m}, 2 \mathrm{~m}}$} & \multirow{2}{*}{$\frac{2.8 \mathrm{~m} / \mathrm{s}}{0.7 \mathrm{~m}, 1.6 \mathrm{~m}}$} \\
\hline & $0.5 \mathrm{~m}, 1 \mathrm{~m}$ & $1 \mathrm{~m}, 1 \mathrm{~m}$ & $0.5 \mathrm{~m}, 2 \mathrm{~m}$ & $1 \mathrm{~m}, 2 \mathrm{~m}$ & & \\
\hline$R_{f}$ & $1493 \times 10^{-6}$ & $654 \times 10^{-6}$ & $1056 \times 10^{-6}$ & & $146 \times 10^{-6}$ & $56 \times 10^{-6}$ \\
\hline$R_{r}$ & $408 \times 10^{-6}$ & $179 \times 10^{-6}$ & $204 \times 10^{-6}$ & $89 \times 10^{-6}$ & $89 \times 10^{-6}$ & $169 \times 10^{-6}$ \\
\hline$R_{v}$ & $181 \times 10^{-6}$ & $89 \times 10^{-6}$ & $90 \times 10^{-6}$ & & $45 \times 10^{-6}$ & $80 \times 10^{-6}$ \\
\hline$R_{c}$ & $786 \times 10^{-6}$ & $393 \times 10^{-6}$ & $452 \times 10^{-6}$ & $226 \times 10^{-6}$ & $226 \times 10^{-6}$ & $386 \times 10^{-6}$ \\
\hline$Q \max$ & $289 \mathrm{~kW}$ & $630 \mathrm{~kW}$ & $460 \mathrm{~kW}$ & $1008 \mathrm{~kW}$ & $1639 \mathrm{~kW}$ & $1202 \mathrm{~kW}$ \\
\hline
\end{tabular}
small for large radius and height of reactor vessel. Several examples of thermal resistances for different radius and height of reactors are summarized in Table 2.

Table 2. Calculation results of thermal resistance and maximum thermal power.

Qmax is the maximum thermal power of the reactor satisfying temperature of fuel salt smaller than its melting point at each condition. Thickness of peripheral reflector and reactor vessel are kept the same at $0.1 \mathrm{~m}$ and $0.01 \mathrm{~m}$, respectively.

Thermal resistance of fuel salt depends on its heat transfer coefficient. The value is determined by $\mathrm{Nu}$ and it increases by superficial velocity. Thus, thermal resistance is evaluated for a large superficial velocity of fuel salt. Qmax for a $1 \mathrm{~m}$ radius, $2 \mathrm{~m}$ height of reactor vessel and $1 \mathrm{~m} / \mathrm{s}$ of superficial fuel salt velocity becomes $1639 \mathrm{~kW}(1.6 \mathrm{MW})$. Qmax for a $0.7 \mathrm{~m}$ radius, $1.6 \mathrm{~m}$ height of reactor vessel and 
$2.8 \mathrm{~m} / \mathrm{s}$ of superficial fuel salt velocity is $1.2 \mathrm{MW}$. These values are of MSRE which operated at ORNL in 1960's. It can be seen that the upper limit of thermal power of presently proposed reactor is small compared to the MSRE's thermal output designed to be $10 \mathrm{MW}$.

This design's policy is to achieve smallness of capacity and ease in manufacturing. Though theoretically the economy of molten-salt reactor is excellent, it has to be confirmed for our new design including additional requirements for fuel preparation, reprocessing and so on.

\subsection{External Fissile Supply by Using Accelerator Named "Mitrailleuse"}

As mentioned in the previous section, this new design does not focus on achieving a high conversion ratio. On the other hand, fissile supply is an essential requirement for utilizing nuclear fission reaction as energy supply. This is more serious for the case of using thorium. By taking account of this requirement, the author also has a research project to provide fissile externally from fission reactor. Fissile can be obtained by exposing neutrons to fertile material. For generating neutrons, an accelerator is available. Recent activities on accelerator driven sub-critical system (ADS) are based on the spallation reaction which occurs when high energy charged proton up to $1 \mathrm{GeV}$ [30]. The authors' proposal is to utilize $\mathrm{D}$-Be reaction for generating neutrons. For this purpose, we have developed a new accelerator named "Mitrailleuse". Details can be found in a previously presented paper [31].

\section{Conclusions}

Recent progress and the future vision of nuclear power are described from the viewpoint of sustainability. It can be said that nuclear power will still play an important role for achieving sustainability. However, there are additional efforts for reducing workload of radioactive waste, improving safety and enhancing nuclear proliferation resistivity of nuclear power. Thorium utilization is considered as a countermeasure. Thorium does not produce a lot of transuranic nuclide and high gamma activity resists nuclear proliferation. Thorium utilization also helps support low carbon automobiles such as electric vehicles or hybrid cars by internalizing the thorium as waste of rare earth production. This is another aspect required from sustainability. The most effective use of thorium is as nuclear fuel. However, it is estimated that more than one decade will be necessary for commercialization of thorium nuclear power. Therefore, it is necessary to store thorium in an international framework. Proposal of "The Bank" by the author corresponds to this. Supply of human resource is also an important task for a sustaining use of nuclear power. Skoda reported that the Czech Republic is developing a thorium molten-salt reactor for the young generation at the IAEA's conference in January 2010 [32].

Molten-salt reactors are reported as highly improving the safety of nuclear power. Though molten-salt reactors essentially offer inherent safety features, there are still many practical matters in the manufacturing and operating of the reactor which are mainly conducted by the outer circulating fuel via primary circuit. The proposal of "internal circulation and a surface heat transferring molten-salt reactor" overcomes these remaining matters. This design is suitable for a small reactor enabling close placement at demand side with high safety. Thus, the balance between benefit and risk can be solved. Lack of fissile material will be externally fed by using a neutron accelerator. 


\section{Acknowledgments}

This work was supported by KAKENHI (2171015 2) for Grant-in-Aid for Young Scientists (B).

\section{Conflict of Interest}

The authors declare no conflict of interest.

\section{References}

1. Brundtland, G.H. Report of the World Commission on Environment and Development: Our Common Future; United Nations: Oslo, Norway, 1987. Available online: http://www.undocuments.net/our-common-future.pdf (accessed on 30 June 2012).

2. International Energy Agency (IEA). World Energy Outlook; IEA: Paris, France, 2009.

3. European Commission. ExternE 1999: Externality of Energy; European Commission, Directorate General XII, Science, Research \& Development: Luxembourg, Luxembourg, 1999.

4. Pigou, A.C. The Economics of Welfare, 4th ed.; Macmillan and Co.: London, UK, 1932.

5. Nuclear Security Summit. Available online: http:/www.state.gov/t/isn/nuclearsecuritysummit/ index.htm (accessed on 30 June 2012).

6. Palme, O. Statement by Prime Minister Olof Palme of Sweden, on the occasion of the commemoration of the Fortieth Anniversary of the United Nations, 21 October 1985, unpublished work.

7. Kamei, T. Symbiotic energy demand and supply system based on collaboration between rare-earth and thorium utilization. Int. Electr. J. Nuclear Saf. Simul. 2011, 2, 178-190.

8. Kamei, T. Study of nuclear environment \& material strategy. Int. Electr. J. Nuclear Saf. Simul. 2011, 2, 339-349.

9. Kamei, T. The Indispensable Role of Thorium for Creating a Sustainable Society. In Proceedings of The 18th Pacific Basin Nuclear Conference (PBNC 2012), BEXCO, Busan, Korea, 18-23 March 2012.

10. Kamei, T. Preliminary Study of OREEC (Organization of Rare-Earth Exportation Countries). In Proceedings of the Conference on Sustainable Development of Energy, Water, and Environment Systems, Ohrid, Macedonia, 1-6 July 2012.

11. Yamawaki, M.; Yamana, H.; Unesaki, H.; Fukuda, K. Recent research trends of thorium fuel cycle (in Japanese). J. At. Energy Soc. Jpn. 2005, 47, 14-33.

12. Moir, R.W. Cost of electricity from molten salt reactors (MSR). Nuclear Technol. 2002, 138, 93-95.

13. IAEA. IAEA Safeguards Glossary 2001 Edition. International Nuclear Verification Series No. 3; IAEA: Vienna, Austria, 2002.

14. Kang, J.; Hippel, F.N. U-232 and the proliferation resistance of U-233 in spent fuel. Sci. Glob. Secur. 2001, 9, 1-32.

15. Furukawa, K.; Arakawa, K.; Erbay, L.B.; Ito, Y.; Kato, Y.; Kiyavitskaya, H.; Lecocq, A.; Mitachi, K.; Moir, R.; Numata, H.; et al. A road map for the realization of global-scale thorium breeding fuel cycle by single molten-fluoride flow. Energy Convers. Manag. 2008, 49, 1832-1848. 
16. NHK ETV Special. "Never-ending" project-A road to nuclear fuel cycle. Available online: http://www.nhk.or.jp/etv21c/file/2012/0617.html (accessed on 30 June 2012).

17. Kamei, T.; Kato, Y.; Mitachi, K.; Shimazu, Y.; Yoshioka, R.; Furukawa, K. Thorium Molten-Salt Nuclear Energy Synergetics for the Huge Size Fission Industry. In Proceeding of the 4th Topical Meeting on Advances in Nuclear Fuel Management 2009 (ANFM IV), Hilton Head Island, CA, USA, 12-15 April 2009.

18. Kamei, T. Implementation Strategy of Thorium Fuel Cycle. In Proceedings of Physor 2010-Advances in Reactor Physics to Power the Nuclear Renaissance, Pittsburgh, PA, USA, 9-14 May 2010.

19. Tsvetkov, P.V. Nuclear Power-Deployment, Operation and Sustainability; InTech: Rijeka, Croatia, 2011; pp. 365-382.

20. Chidambaram, R. Human Resource Development: Nuclear Renaissance and Sustainable Energy Security. In Proceedings of the International Conference on Human Resource Development for Introducing and Expanding Nuclear Power Programmes, Abu Dhabi, United Arab Emirates, 14-18 March 2010.

21. Roy, C.; Robertson, C. Conceptual Design Study of a Single-Fluid Molten-Salt Breeder Reactor. ORNL-4541; U.S. Atomic Energy Commission: Washington, DC, USA, 1971.

22. Kamei, T. Thorium MSR as a Small-Scale Energy Source-Opportunities for Japan. In Proceedings of the ASME 2011 Small Modular Reactors Symposium SMR2011, Washington, DC, USA, 28-30 September 2011.

23. Renault, C.; Delpech, M. The Most Project: Key-Points and Challenges for the Feasibility of Molten Salt Reactors. In Proceedings of the ICAPP'05, Seoul, Korea, 15-19 May 2005.

24. Idaho National Laboratory. Generation IV Nuclear Energy Systems Ten-Year Program Plan Fiscal Year 2007; U.S. Department of Energy: Washington, DC, USA; Volume I.

25. Generation IV International Forum. The Molten Salt Reactor (MSR) system produces fission power from a molten salt fuel circulating in a fast or epithermal-spectrum reactor and contains an integrated fuel cycle. Available online: http:/www.gen-4.org/Technology/systems/msr.htm (accessed on 30 June 2012).

26. Hirose, Y. Feasibility and Use of Fluoride Molten-Salt Reactors for the TRU Incineration. In Proceedings of the 2012 Annual Meeting of the Atomic Energy Society of Japan, Fukui, Japan, 19-21 March 2012.

27. Merle-Lucotte, E. Launching the Thorium Fuel Cycle with the Molten Salt Fast Reactor. In Proceedings of the ICAPP 2011, Nice, France, 2-5 May 2011.

28. Ignatiev, V.; Feynberg, O.; Myasnikov, A.; Zakirov, R. Reactor Physics \& Fuel Cycle Analysis of a Molten Salt Advanced Reactor Transmuter. In Proceeding of International Conference on Advanced Power Plants, ICAPP '03, Cordoba, Spain, 4-7 May 2003.

29. Yamamoto, T.; Mitachi, K.; Nishio, M. Unsteady Characteristics of Three-Core Molten Slat Reactor-A Study on Load-Following Capability. Therm. Sci. Eng. 2007, 15, 75-84.

30. Aït Abderrahim, H.; Baeten, P.; De Bruyn, D.; Heyse, J.; Schuurmans, P.; Wagemans, J. MYRRHA, a multipurpose hybrid research reactor for high-end applications. Nuclear Phys. News 2010, 20, 24-28. 
31. Kokubo, Y.; Kamei, T. Conceptual Design of Thorium-Fuelled Mitrailleuse Accelerator-Driven Subcritical Reactor Using D-Be Neutron Source. In Proceedings of the PHYSOR2012, Knoxville, TX, USA, 15-20 April 2012.

32. Skoda, R. Nuclear Power and New Generation: Attractive Profession? In Proceedings of the International Conference on Human Resource Development for Introducing and Expanding Nuclear Power Programmes, Abu Dhabi, United Arab Emirates, 14-18 March 2010.

(C) 2012 by the authors; licensee MDPI, Basel, Switzerland. This article is an open access article distributed under the terms and conditions of the Creative Commons Attribution license (http://creativecommons.org/licenses/by/3.0/). 